\title{
RELAXED STATES OF MHD TURBULENCE: MINIMUM DISSIPATION OR MINIMUM ENERGY?
}

\author{
David Montgomery \\ Department of Physics \& Astronomy \\ Dartmouth College \\ Hanover, NH 03755, USA
}

\begin{abstract}
Driven, dissipative MHD fluids often seem to undergo relaxation processes. After a turbulent formation phase, a geometrically simpler and less disordered configuration emerges. The best known example is the laboratory reversed-field pinch (RFP); similar field topologies have been proposed for solar prominences and astrophysical "flux ropes." In a transient situation, the more rapid decay of kinetic and magnetic energy relative to magnetic helicity provides a mechanism for generating an MHD configuration with several similarities to observed RFP states. (This is the Tayior hypothesis, not unrelated to turbulent inverse magnetic cascades.) For the driven steady state, however, all quantities are supplied at the same time-averaged rate at which they are dissipated, by definition; nothing decays relative to anything else. Some other unifying principle, beyond "minimum energy" or "selective decay," seems necessary to describe the results of driven, steady-state MHD computations. We have been attempting to adapt the principle of minimum energy dissipation rate to MHD. It is a 19th century principle that achieved some success in hydrodynamics and separately in dissipative electrodynamics.
\end{abstract}

Suppose that a particle is trapped around the minimum of a potential well and at the same time experiences a velocity-dependent frictional energy loss. Eventually, the particle will come to rest at the minimum of the well with zero kinetic energy. This is the simplest example of a "minimum energy state," established subject to a constraint.

Minimum energy states, in more elaborate guises, appear all over physics. States of minimum energy, subject to constraints and boundary conditions, have played a role in ideal magnetohydrodynamic (MHD) stability theory. More recently, Taylor [1,2] has argued that the laboratory toroidal $Z$ pinch ("screw pinch") should relax to a state of minimum energy compatible with approximate conservation of magnetic helicity. Minimum energy states have also been used as a basis for describing solar prominences.

Some physical justification for relaxation to states of minimum energy can be given for MHD in the context of the decaying initial value problem for isolated systems. The arguments involve the more rapid turbulent decay of energy, relative to magnetic helicity, or "cross" helicity, or whatever other ideal invariants might decay less rapidly because of

E. R. Priest and V. Krishan (eds.), Basic Plasma Processes on the Sun, 215-222.

(C) 1990 IAU. Printed in the Netherlands. 
tendencies to being transferred preferentially to the long-wavelength end of the spectrum [3-8].

However, the minimum energy formulation seems to be useful mainly for discussing decaying initial-value situaliuns. Iis implications come to seem doubtiul in diven, steadystate situations in which the excitation of the system is balanced by dissination. To take the example again of a particle trapped in a potential well, it will clearly r st settle into its minimum energy state if it is continually acted on by some external for.e. Rather, it will stay on the average at some finite level above its minimum possible energy at a place where its rate of absorption of energy from the external force will be on the average balanced by its rate of frictional energy loss. It cannot go to a "minimum energy" state.

Driven, dissipative conditions are often closer to the realities of high-Reynolds-number or high-Lundquist-number MHD fluids than is the model of initially-excited, isolated decay. This is true both in the laboratory and on the sun. Driving mechanisms can be voltage drops, pressure drops, or mechanical agitation such as thermal convection. The dissipation is resistive and viscous. The question arises as to whether there is a variational principle that is as useful for the driven, steady-state problem as the minimum energy principle is for isolated mechanical systems, particularly for fluids and magnetofluids.

We are not completely sure of the answer, but we think there is. It is the principle of minimum energy dissipation rate. It seems to have been first formulated by Kirchoff, who asked the following question.

Given a rigid electrical conductor of fixed non-uniform electrical conductivity $\sigma(\mathbf{x})$ with the electrostatic potential $\Phi(\mathbf{x})$ fixed over a closed surface, how will the electric current deñoity $\mathbf{j}(\boldsymbol{x})$ distrituute itself inside the surface? The equation of continuity reiates the electric charge density $\rho_{e}$ to $\mathrm{j}$ :

$$
\frac{\partial \rho_{e}}{\partial t}+\nabla \cdot \mathbf{j}=0
$$

For either a steady state or a time average, the $\partial \rho_{e} / \partial t$ drops out and we are left with $\nabla \cdot \mathbf{j}=0$. However, $\mathbf{j}=-\sigma(\mathbf{x}) \nabla \Phi(\mathbf{x})$. Making this substitution, the differential equation

$$
\nabla \cdot(\sigma(\mathbf{x}) \nabla \Phi(\mathbf{x}))=0
$$

then determines $\Phi(\mathbf{x})$ and $\mathbf{j}(\mathbf{x})$, given $\Phi(\mathbf{x})$ over the surface. Notice that Eq. (2) is exactly the Euler-Lagrange equation that one would have gotten if one had set out to minimize the total Ohmic energy dissipation rate $R_{j}$,

$$
R_{j} \equiv \int \sigma^{-1} \mathbf{j}^{2} d^{3} x=\int \sigma(\nabla \Phi)^{2} d^{3} x
$$

where the integral runs over the volume interior to the surface over which $\Phi$ is given.

The answer to Kirchoff's question is that $\mathbf{j}(\mathbf{x})$ arranges itself in such a way as to minimize the energy dissipation rate. Note that the given constrained value of $\Phi(\mathbf{x})$ over the boundary is the constraint that gives individual cases their content. This is essentially a rigorous proof.

Somewhat less rigorous, but still correct, is the application of the minimum dissipation rate principle to viscous fluid flows in hydrodynamics [11] made later by Helmholtz, Korteweg, and Lord Rayleigh. If one minimizes the viscous energy dissipation rate $R_{\omega}$,

$$
R_{\omega} \equiv \int \mu \omega^{2} d^{3} x=\rho_{0} \int \nu \omega^{2} d^{3} x
$$


where $\omega=\nabla \times v$ is the vorticity of a uniform incompressible fluid, $\mu$ is its viscosity, and $\rho_{0}$ and $\nu$ are its density and kinematic viscosity, one is quickly led to the elementary steady flows. Plane Poiseuille flow, pipe flow, plane Couette flow, rotating Couette flow are all derivatule by iminimizing $\boldsymbol{n}_{\boldsymbol{\omega}}$ subjeci io ihe relevant boundary conditions. (For the case of moving boundaries, $R_{\omega}$ differs from the dissipation rate expressed as an integral involving the rate-of-strain tensor by an integral whose first variation vanishes $f c:$ trial functions $\delta \mathbf{v}(\mathbf{x})$ which vanish on the boundary, so that minimizing $R_{\omega}$ is still mini.nizing the energy dissipation rate.) As in the electric current case, it is the boundary ronstraints that give individual cases their content.

It came to seem natural to us to re-examine driven, dissipative MHD from the point of view of minimum-dissipation-rate principles. In a familiar set of dimensionless variables, the relevant integral to be minimized for incompressible, resistive, viscous MHD is $\left(\eta=\sigma^{-1}\right)$

$$
R=R_{\omega}+R_{j}=\int\left(\nu \omega^{2}+\eta \mathbf{j}^{2}\right) d^{3} x
$$

subject to appropriate constraints. We have been particularly interested [12-15] in the case of voltage-driven cylinders of magnetically-supported MHD fluid with periodically-identified ends (to simulate toroidal periodicity). This situation is one for which extensive numerical computations due to Dahlburg, et al. [16-18] and Theobald, et al. [19] have for some time been accumulating evidence that only in some respects did minimum-energy predictions seem to represent what happened, and that a fundamental revision was in order.

Noteworthy among the previuusiy unexplained features, that conid not he squared with the minimum energy predictions, were: (1) a strong spatial dependence of the ratio $|\mathbf{j}| /|\mathbf{B}|$ (here, $\mathbf{B}$ is the magnetic field and $\mathbf{j}=\nabla \times B$ in the relaxed, approximately force-free state); (2) a persistent helical contribution to $j$ and $B$, in addition to the (larger) approximately axisymmetric components; and (3) a persistent kinetic energy of flow, at the level of a few percent, also tending to be helically distributed over space. These results had been around for some time before we found any way to get at them analytically.

The principal difficulty in minimizing the dissipation rate $R$ (or, for that matter, in minimizing the energy, if anyone still feels that that is what he should do) is the role played by the Ohm's law, which for MHD can be written in the equivalent forms

$$
\frac{\partial \mathbf{B}}{\partial t}=\nabla \times[\mathbf{v} \times \mathbf{B}-\eta \mathbf{j}]
$$

or

$$
\mathbf{E}=\mathbf{E}_{\mathbf{0}}+\nabla \tilde{\boldsymbol{\Phi}}=-\mathbf{v} \times \mathbf{B}+\eta \mathbf{j}
$$

Here, $\mathbf{E}$ is the (dimensionless) electric field, $\mathbf{E}_{0}$ is its spatial average, $\tilde{\boldsymbol{\Phi}}$ is a scalar potential determined by

$$
\nabla^{2} \tilde{\boldsymbol{\Phi}}=-\nabla \cdot(\mathbf{v} \times \mathbf{B})
$$

if the resistivity $\eta$ is spatially uniform.

Eq. (6), or (7), is the source of whatever constraints apply to this system beyond simple boundary conditions, including all those involving global magnetic helicity or its 
rate of supply. But it is much more than that. Since $\tilde{\Phi}=\tilde{\Phi}(\mathbf{v}, \mathbf{B})$ as a consequence of Eq. (8), Eq. (7) is in effect a pointwise constraint relating $\mathbf{v}, \mathbf{B}$ and $\nabla \times \mathbf{B}$ at every point in space. Its complexity precludes elimination of either $\mathbf{v}$ or $\mathbf{B}$ in favor of the other ânủ substitution intu $\boldsymbol{R}$. Most imporiantìy, Eq. (i) mandares a ninite fiow velocity $\mathbf{v}$ for all states except the relatively uninteresting one of uniform $\mathbf{j}=\mathbf{E}_{\mathbf{0}} / \eta$. This $\mathbf{v}$ is not the fancy, statistically-distributed small-scale turbulence invoked in the "alrha effect," it is macroscopic and present in the simplest cases. It requires a sort of zerot!1-order revision in our ideas of what an MHD equilibrium can be. It may devalue, forever, the ideal, cylindrical equilibria with zero-flow, axisymmetric current distributions $\mathbf{j}=\left(0, j_{\varphi}(r), j_{z}(r)\right)$ that have been such a preoccupation in much of MHD.

If one takes the hydrodynamic point of view that ideal equilibria are only physically significant if they can be approached as a limit, as the small but finite dissipation coefficients approach zero, then Ohm's law calls into question most of the ideal axisymmetric zero-flow equilibria that have been proposed. It turns out to be rather difficult to construct resistive MHD equilibria which obey not only force balance according to the equation of motion, but also flux balance as required by Eqs. (6) and (7), with small but non-zero $\eta$. The reason, not at all unfamiliar in hydrodynamics, is that $\eta$ multiplies the highest spatial derivative of $\mathrm{B}$ in the problem.

It has been possible [13-15] to construct some solutions to the full set of MHD equations which obey Eqs. (6) and (7) as well as the $\partial \mathbf{v} / \partial t$ equation with viscosity. The solutions so far are perturbation-theoretic. The small parameier is ine raiio of helical to the axisymmetric parts of $\mathbf{j}$ and $\mathbf{B}$. The solutions go through second order in this ratio. Graphically [13], they look in many respects like the results of the MHD computations [16-19] in their principal features. The agreement is better at not too high values of the pinch ratio $\Theta$, as might be expected. The analytical solutions, like the numerical computations, are lengthy, and it is not our purpose to reproduce them here, but to direct attention to the original papers. Further extensions, including predictions of the amplitudes of the helical components, will be reported elsewhere [20].

\section{Discussion}

What are the implications of these considerations for solar physics? Perhaps their immediate impact is not great, since the quantities being revised, such as the aforementioned helical $\mathbf{v}$ and $\mathbf{B}$ contributions, are likely out of reach observationally in the solar context in the immediate future. Perhaps of more value is a reminder of how little of what passes for magnetohydrodynamic theory is really on very solid ground. In all astrophysics, there is a tendency to concentrate on the observations and to regard MHD as a consumer regards an off-the-shelf product. Like all consumers, the observer would like to believe the product he is buying has been thoroughly tested and is reliable. Nobody wants to hear that the propositions of MHD theory, which have been repeated so many times, are shaky ones about which the buyer had better beware. But that is, probably, the case. It did not need to be this way. For a small fraction of the expense of the many observations, a little investment in internally-diagnosable laboratory MHD would have left us far ahead of where we are at 
present. We would at least know whether or not to expect flows in pinch situations!

\section{Acknowledgements}

This work was supported in part by NASA Grant NAG-W-710 and U.S. Department of Energy Grant DE-FG02-85ER53194.

\section{References}

1. Taylor, J.B. (1974) Relaxation of toroidal plasmas and generation of reverse magnetic fields, Phys. Rev. Lett., 33, 1139.

2. Taylor, J.B. (1986) Relaxation and magnetic reconnection in plasmas, Revs. Mod. Phys. 58, 741.

3. Montgomery, D., Turner, L. and Vahala, G. (1978) Three-dimensional MHD turbulence in cylindrical geometry, Phys. Fluids, 21, 757.

4. Matthaeus, W.H. and Montgomery, D. (1980) Selective decay hypothesis at high mechanical and magnetic Reynolds numbers, Proc. Int. Conf. on Nonlinear Dynamics, Ann. NY Acad. Sci., 357, 203.

5. Riyopoulos, S., Bondeson, A. and Montgomery, D. (1982) Relaxation toward states of minimum energy in a compact torus, Phys. Fluids, 25, 107.

6. Ting, A.C., Matthaeus, W.H. and Montgomery, D. (1986) Turbulent relaxation processes in magnetohydrodynamics, Phys. Fluids, 29, 3261.

7. Dahlburg, J.P., Montgomery, D., Doolen, G.D. and Turner, L. (1987) Turbulent relaxation of a confined magnetofluid to a force-free state, J. Plasma Phys., 37, 299.

8. Montgomery, D. and Phillips, L. (1989) MHD turbulence, relaxation processes and variational principles, Physica, D37, 215.

9. Kirchoff, G.D. (1848) in Ann. Phys., 75, 189.

10. Jaynes, E.T. (1980) The minimum entropy production principle, Ann. Rev. Phys. Chem., 31, 579.

11. Lamb, H. (1945) Hydrodynamics, 6th ed., Dover, New York, pp. 617-619.

12. Montgomery, D. and Phillips, L. (1988) Minimum dissipation rates in magnetohydrodynamics, Phys. Rev. A38, 2953.

13. Montgomery, D., Phillips, L. and Theobald, M.L. (1989) Helical, dissipative, magnetohydrodynamic states with flow, Phys. Rev., A40, 1515.

14. Montgomery, D. (1989) Relaxed states in driven, dissipative magnetohydrodynamics: helical distortions and vortex pairs, to appear in Trends in Theoretical Physics, Vol. I, ed. by P.J. Ellis and Y.C. Tang, (Addison-Wesley, New York).

15. Montgomery, D. (1990) Minimum dissipation states and vortical flow in MHD, to appear in Proc. 1989 IUTAM Symposium, H.K. Moffatt, Ed. (Cambridge University press, Cambridge, UK).

16. Dahlburg, J.P., Montgomery, D., Doolen, G.D. and Matthaeus, W.H. (1986) Largescale disruptions in a current-carrying magnetofluid, J. Plasma Phys., 35, 1.

17. Dahlburg, J.P., Montgomery, D., Doolen, G.D. and Turner, L. (1986) Turbulent relaxation to a force-free field-reversed state, Phys. Rev. Lett., 57, 428.

18. Dahlburg, J.P., Montgomery, D., Doolen, G.D. and Turner, L. (1988) Driven steadystate RFP computations, J. Plasma Phys., 40, 39. 
19. Theobald, M.L., Montgomery, D., Doolen, G.D. and Dahlburg, J.P. (1989) Sawtooth oscillations about helical current channels, Phys. Fluids, B1 766.

20. Montgomery, D., Chen, H. and Shan, X, to be published. 


\section{DISCUSSION}

VAN BALLEGOOIJEN: How does the velocity scale with magnetic diffusivity $(\eta)$ ? Is the scaling linear?

MONTGOMERY: We do not really know in any comprehensive sense. There is not any velocity, for example, until the applied electric field $\mathrm{E}_{0}$ crosses a threshold, $\mathrm{E}_{\mathrm{c}}$. Just above that, the rms velocity seems to go linearly with $\eta^{1 / 2}$ but it is also multiplied by $\left(E_{0}-E_{c}\right)$, in the expression we have been able to calculate. $E_{c}$ itself depends on magnetic diffusivity, though, so the answer does not appear to be too simple.

HEYVAERTS: Has it been possible to recognize any sign of intrinsic stochasticity of field lines in your numerical solutions?

MONTGOMERY: No, not a great deal. We have made only a few attempts to track field lines the requisite large number of times around the torus and make Poincare-type plots of their interesections with planes of constant $z$. The results of one such attempt appear in Figs.8 of Phys.Fluids B1, 766 (1989), which to me, at least, look pretty regular. I sometimes feel that the question of "where the field lines go" receives too much emphasis in MHD, mostly for historical reasons. If one is concerned with a population of hot electrons trying to escape from a tokamak, then it is needed information. But if MHD alone is one's concern, all the same information is available in the various fields $(b,(x, t), j(x, t), \mathbf{z}(\mathbf{x}, \mathbf{t})$, $\underline{A}(\mathrm{x}, \mathrm{t})$ etc. as functions of space and time. These are what the computer provides, and it is a relatively straightforward operation to ask it to make 3D perspective plots, contour plots, etc., of these various fields. It is a delicate and risky matter to try to follow a turbulent field line, which is perhaps why people seldom try in hydrodynamics. Also, when resistivity is present, there is no unique way to identify a field line from one time to the next. I would like to see us declare a moratorium on anthropomorphic speculations about field line behaviour.

BUTI: The appearance of chaos in a magnetic field would depend on the strength of the driver. It is possible that the driver you have taken is not strong enough and it is possible that you would see the chaos if you take a stronger driver.

MONTGOMERY: I guess by "chaos in the magnetic field" one might mean two things: (i) chaotic Poincaré plots of magnetic field intersections with a plane of constant $z$ at a fixed time; or (ii) chaotic, Lorenz-like, temporal behaviour in the computed expansion coefficients for the magnetic field. Let me remark on these separately.

(i) In Phys. Fluids,B1,766 (1989) we tried to follow field lines at fixed times and make plots of their successive intersections. In those plots (which refer to the "tokamak" regime), we could see relatively little indication of "chaos". Chaotic patterns may be more likely in the "RFP" regime. It is not easy to design a field-line-tracing algorithm that will follow a field line very many times around a torus reliably, in the presence of genuine 3D 
turbulence. When one of the components is locked away from zero, as in the Strauss approximation, it is somewhat easier. But the turbulence is also somewhat suppressed, perhaps artificially, by the approximation.

(2) In J. Plasma Phys. 35,1 (1986), a very low-order truncation of the dynamical equations in transform space did, when numerically integrated, yield Lorenz-like, "chaotic" behaviour in the phase space. However, as more terms in the expansion were added, and the truncation became less severe, the exotic "strange attractor" behaviour went away.

VENKATAKRISHNAN: How does the kinetic energy of final relaxed state scale with the energy input at the boundaries?

MONTGOMERY: Energy input is something that, unlike magnetic helicity input, is not fixed by the boundary conditions we are imposing. It is up to the plasma, which behaves as an anomalously resistive element due to the $\langle\underline{v} \times \underline{B}\rangle$ opposing the applied electric field, and the amount of energy it accepts is not fixed by prescribing the electric field at the wall. Roughly speaking, the two quantities go up together, but their basic dependence on a prescribable number is better found by asking how both go up as a function of applied electric field at the wall, or "toroidal loop voltage". We plan to give an answer to that for the "tokamak regime" (pinch ratio $\Theta<1$ ) in an upcoming manuscript.

KRISHAN: You mentioned that the hydrodynamicists made a mistake by neglecting viscosity. What are the consequencies of including viscosity?

MONTGOMERY: There is not much similarity, in hydrodynamics, between the case of zero viscosity and very small viscosity; it is a kind of discontinuous limit. When the Reynolds' number becomes $>1000$ or 2000 , turbulence sets in in the presence of velocity shear. The instabilities which signal it are intrinsically viscous. Also, since viscosity (like resistivity in MHD) multiplies the highest-order spatial derivatives in the problem, its neglect changes in fundamental ways the number and kind of boundary conditions required for a well-posed problem. There is absolutely no guarantee that, if a zero-viscosity solution can be found, there is a similar solution with small but finite viscosity. More often than not, there is not.

HOLLWEG: (i) What do you think the effects of line-tying boundary conditions will be?

(ii) What is the "Alfvénicity" of the final relaxed state?

MONTGOMERY: (i) This is pretty hard to say. For the "line-tied" case, in which the magnetic field lines intersect the conducting boundary, a lot more needs to be said in order to feel confident that we have a set of boundary conditions that will determine a well-posed problem.

(ii) The ratio of kinetic energy of motion to magnetic energy per unit volume is always small for the case we have mostly considered, where there are rigid, conducting boundaries and the driving is through applied voltages. Typical numbers would be two to four per cent. In the presence of these highly "magnetic" boundary conditions, the kinetic energy gets clamped at a fairly low level, and the tendencies toward equipartition at the larger scales that might be expected for periodic boundary conditions and no imposed dc magnetic field just do not appear. 